\title{
Dual-targeting decoy
}

\section{By Kai-Jye Lou, Staff Writer}

Although inhibitors of VEGF or tumor necrosis factor- $\alpha$ are among the most successful drugs on the market, researchers at the Korea Advanced Institute of Science and Technology think they can go one better by blocking both targets with a single molecule. The team has proof of principle with a dual-targeting decoy receptor that had better efficacy than antiVEGF candidate aflibercept and anti-tumor necrosis factor drug Enbrel etanercept combined in mouse models of retinopathy and psoriasis. ${ }^{1}$

"Whenever we examined patients with retinopathies and some severe and chronic allergic inflammatory diseases like psoriasis, we found that levels of VEGF-A and TNF- $\alpha$ in patient samples were concomitantly high," said Gou Young Koh, a professor in the graduate school of biomedical science and engineering at KAIST. "Therefore, we hypothesized that if we blocked these two molecules simultaneously, we could treat such diseases more efficiently" than hitting either target alone.

The KAIST researchers used a double-decoy trap technology ${ }^{2}$ to engineer a decoy receptor, dubbed Valpha. It contains the VEGF-A-binding domain of VEGF receptor 1 (FLT1), the tumor

"The advantage of giving a single molecule that goes after two targets is that you will only have one biodistribution profile."

-Gou Young Koh,

Korea Advanced Institute of Science and Technology

to the Fc portion of human IgG1 (TNFr:Fc), for multiple autoimmune diseases including psoriasis and RA.

Aflibercept (VEGF Trap) is in Phase III testing for multiple cancers. The fusion protein containing the extracellular domains from two VEGF receptors linked to the Fc portion of human IgG is being developed by Regeneron Pharmaceuticals Inc. and partner sanofi-aventis Group. VEGF Trap-Eye aflibercept ophthalmic solution, which Regeneron is developing with partner Bayer AG, is under FDA review for wet AMD.

Amgen and Regeneron declined to comment.

\section{Dual-targeting dilemmas}

"The Valpha molecule described in this $J B C$ paper is interesting in that it suppresses both TNF- $\alpha$, a well-validated inflammatory cytokine, and VEGF-A, a factor required to supply the high metabolic requirements of chronically inflamed tissues with new vasculature," said Brian Wong, VP of immunology and discovery research at Five Prime Therapeutics Inc., which develops protein therapeutics for oncology, immunology and metabolic indications. "In theory, this approach should be more efficacious than targeting either TNF- $\alpha$ or VEGF-A alone."

"The advantage of giving a single molecule that goes after two targets is that you will only have one biodistribution profile," said Koh, who is corresponding author on the paper. "In contrast, if you use a combined treatment, each compound will have its own biodistribution profile and thus may not reach the desired microenvironment simultaneously to have a synergistic effect." necrosis factor- $\alpha$ (TNF- $\alpha$ )-binding domain of tumor necrosis factor receptor 2 (TNFR2) and the Fc domain of IgG1. In vitro characterization showed that Valpha bound strongly to VEGF-A and TNF- $\alpha$ with dissociation constants $\left(K_{\mathrm{d}}\right)$ of about $6.5 \mathrm{pM}$ and $64.1 \mathrm{nM}$, respectively.

In a mouse model of psoriasis, Valpha did a better job than Enbrel plus aflibercept in attenuating disease-associated edema and angiogenesis.

In a mouse model of age-related macular degeneration (AMD) and diabetic retinopathy, Valpha was more effective at lowering macrophage infiltration to the retina than combination treatment with Enbrel plus aflibercept. Macrophage infiltration is a marker of injury and diseaseassociated inflammation.

Valpha did not best Enbrel and aflibercept on all AMD measurements. The KAIST molecule produced decreases in disease-associated neovascularization that were comparable to those created by the combination. Taken with the prior result, the findings suggest that if Valpha is ultimately found to be more effective in the clinic, it will likely be due to its stronger anti-inflammatory effect as opposed to its antiangiogenic effect.

However, in a mouse model of rheumatoid arthritis (RA), Valpha produced reductions in disease severity that were comparable to those produced by Enbrel or aflibercept alone, which suggests the combination would not provide added benefit in RA.

Results were published in The Journal of Biological Chemistry.

Amgen Inc. markets Enbrel, a recombinant p75 TNF receptor linked
According to Koh, the binding of Valpha to one of its targets may increase the decoy receptor's ability to bind to its other target. He said this phenomenon, known as the avidity effect, also could contribute to Valpha's improved efficacy over the combination of single-targeting agents in the retinopathy and psoriasis mouse models.

Valpha could have convenience and cost advantages over administering a pair of single-targeting biologics, Koh added.

Ryo Kubota, president and CEO of Acucela Inc., suggested that "this approach has potential in the treatment of wet AMD and may also be useful in dry AMD."

In wet $\mathrm{AMD}$, abnormal blood vessel growth damages the macula and leads to vision loss. Vision loss in dry AMD is caused by atrophy of the retinal pigment epithelium (RPE) in the eye, which supplies photoreceptors with nutrients.

Acucela's ACU-4429, a small molecule that targets the RPE-specific protein $65 \mathrm{kDa}$ (RPE65) to modulate the visual cycle, is in Phase II testing to treat dry AMD.

Despite the advantages of simultaneously attacking two disease targets with a single compound, Wong expects there will be trade-offs compared with single-targeting compounds.

"Bispecific or multispecific proteins would be expected to be larger and more complex molecules, which could translate into increased cost of goods as well as an increased risk of immunogenicity," he told SciBX. 


\section{ANALYSIS}

\section{TARGETS \& MECHANISMS}

"Another disadvantage is not being able to easily control the relative amounts of each targeting component as you could with two separate molecules. This may make it more difficult to optimize dosing for optimal efficacy and safety."

Koh said his group has not found any detectable immunogenicity in preclinical studies but is planning to carry out a more comprehensive and long-term set of studies in animal models to confirm this.

Koh also noted that the complexity and cost of manufacturing Valpha is comparable with those of single-targeting agents like aflibercept and Enbrel. He added that his group is trying to optimize and scale up the product's manufacturing process.

"We currently have a $\mathrm{CHO}$ cell line that produces Valpha at a rate of about one gram per liter, but we are hoping to generate a cell line that could produce Valpha at a rate of two grams per liter," he said. "In our case, getting more than one gram of Valpha per liter in CHO cells is good enough for commercial-scale production."

\section{Choice indications}

Koh said his group is seeking an industry partner to help accelerate Valpha's development and thinks it would be best to first evaluate the decoy receptor in severe diabetic retinopathy or AMD. He thinks Valpha could be ready for Phase I testing in three to four years if the group is able to find a partner this year.

"More than half of the cases of diabetic retinopathy and age-related macular degeneration are accompanied by severe inflammation that is caused by upregulation of inflammatory cytokines like TNF- $\alpha$," Koh said. "Moreover, Valpha should be competitive in terms of cost and efficacy in these indications."
Acucela's Kubota said the use of Valpha in dry AMD may need additional testing in animal models to clearly show that the decoy receptor has a protective effect on the RPE.

In addition, both Wong and Kubota think additional dose-response studies in animal models are needed to help determine whether Valpha can be given at levels that are considered safe and effective for a particular indication in patients.

"We will need to be more cautious when trying to move Valpha into the clinic for psoriasis as there are currently no ideal animal models for this disease," Koh noted.

He said his group has no plans to develop Valpha in arthritis.

Valpha is covered by a pending patent from KAIST. The compound is available for licensing.

Lou, K.-J. SciBX 4(13); doi:10.1038/scibx.2011.358

Published online March 31, 2011

\section{REFERENCES}

1. Jung, K. et al. J. Biol. Chem.; published online Feb. 23, 2011; doi:10.1074/ jbc.M111.228130

Contact: Gou Young Koh, Korea Advanced Institute of Science and Technology, Daejeon, South Korea

e-mail: gykoh@kaist.ac.kr

2. Koh, Y.J. et al. Cancer Cell 18, 171-184 (2010)

COMPANIES AND INSTITUTIONS MENTIONED

Acucela Inc., Seattle, Wash.

Amgen Inc. (NASDAQ:AMGN), Thousand Oaks, Calif.

Bayer AG (Xetra:BAY), Leverkusen, Germany

Five Prime Therapeutics Inc., South San Francisco, Calif.

Korea Advanced Institute of Science and Technology,

Daejeon, South Korea

Regeneron Pharmaceuticals Inc. (NASDAQ:REGN), Tarrytown, N.Y.

sanofi-aventis Group (Euronext:SAN; NYSE:SNY), Paris, France 